\title{
Tidal Disruption Events With An On-Beam Or Off-Beam Relativistic Jet
}

\author{
Wei-Hua Lei ${ }^{*+}$ \\ School of Physics, Huazhong University of Science and Technology, Wuhan 430074, China \\ E-mail: Leiwhahust.edu.cD
}

\begin{abstract}
A star or sub-stellar object will be destroyed by tidal forces when it passes close enough by a supermassive black hole (SMBH). These events known as TDEs are expected to produce luminous flare emission in the UV to X-ray band. The observations of Sw J1644+57, in particular, suggest that at least some TDEs can launch an on-beam relativistic jet. A common speculation is that these rare events are related to rapidly spinning BHs. We constrained the $\mathrm{BH}$ spin parameter by using the available data, and found that the $\mathrm{BH}$ indeed carries a moderate to high spin, suggesting that BH spin is likely the crucial factor behind the Sw J1644+57-like events. Other observational evidences include the rough 2.7 day periodicity in X-ray dips and 200s QPO, which we interpret as due to spin-induced precession. In addition, Sw J2058+05 and Sw J1112.2-8238 are also thought to be a TDE with an on-beam relativistic jet. It is natural to expect that there should be some events with off-beam ones. We found that IGR J12580+0134 in the nucleus of NGC 4845 is likely such a case.
\end{abstract}

High Energy Phenomena in Relativistic Outflows VII - HEPRO VII

9-12 July 2019

Facultat de Física, Universitat de Barcelona, Spain

\footnotetext{
* Speaker.

${ }^{\dagger}$ This work is supported by National Natural Science Foundation of China under grant 11773010.
} 


\section{Introduction}

Supermassive black holes (SMBHs) can capture and tidally disrupt stars or substellar objects orbiting nearby. These events - known as TDEs — are expected to occur every $10^{3}-10^{5}$ years for a typical galaxy [四]. The debris of the stellar object will be accreted onto the black hole (BH), producing flaring emission in X-ray, ultraviolet, and optical. A typical $t^{-5 / 3}$ behavior of the observed luminosity tracking the fallback rate evolution of the stellar debris, is a distinctive feature of TDEs []].

Most previous studies of tidal disruption events (TDEs) from super-massive BHs did not expect an associated relativistic jet [B]. The detection of $\mathrm{Sw} \mathrm{J} 1644+57$ at $z=0.35$ suggested that at least some TDEs can launch a relativistic jet towards Earth, which is manifested as a superEddington X-ray burst [ [ 4 ] and a long lasting radio emission resulting from jet-medium interaction [回]. Invoking the Blandford \& Znajek (1977, BZ) [可] mechanism as the power of the jet, we found that the SMBH in Sw J1644+57 carries a moderate to high spin [ [ $]$. We therefore suggest that BH spin is the key factor behind the Sw J1944+57-like events. For such events, the outer disk is very likely misaligned with respect to the equatorial plane of the spinning SMBH, since the incoming star before disruption most likely has an inclined orbital plane. The tilted disk is subject to the LenseThirring torque, which tends to twist and warp due to the BardeenPetterson effect. The relativistic jet launched from the spinning SMBH would undergo precession. We confirmed that a 2.7 day quasi-periodic signal exists in the 530 day X-ray light curve of Sw J1644+57. A natural mechanism to account for these dips would be a precessing jet [[13].

Sw J2058+05 [ए4] and Sw J1112.2-8238 [ए5] are two other TDE candidates with an on-beam relativistic jet. A direct expectation is that there should be TDE relativistic jets that beam away from Earth. The nearby TDE IGR J12580+0134, discovered in X-rays by Integral and then detected in radio by JVLA, likely launched a relativistic jet. Similar to Sw J1644+57, the X-ray emission shows distinctive behavior at late time compared with the expectation of the external shock synchrotron emission from the jet-CNM interaction, which suggests a non-external-shock origin. Taking Sw $\mathrm{J} 1666+57$ as a template, we find that the expected internal jet emission would outshine the observed flux greatly. This suggests that the jet is off-beam [ए6].

\section{TDE with an on-beam relativistic jet}

A hard X-ray transient event, Sw J1644+57 was discovered by the Swift satellite [प]] on 2011 March 28, initially reported as GRB 110328A. It was soon realized that it is not a regular GRB. The long variability timescale t $100 \mathrm{~s}$ [䧃] and its location near the center of a $\mathrm{z}=0.354$ host galaxy [ए8] link Sw J1644+57 to a SMBH with $M_{\bullet} \sim 10^{7} M_{\odot}$. The sharp onset and gradual fade away of $\mathrm{X}$-ray flux refer to tidal disruption of a star by a dormant SMBH [⿴囗⿴囗十). The super-Eddington X-ray luminosity [团]), bright radio afterglow [[]], as well as a stringent historical X-ray flux upper limit suggest that a relativistic jet is launched from an SMBH during the TDE, which was not expected in most previous TDE studies [B]].

Modeling the emission of Sw J1644+57 suggests that the jet is highly particle starved [四], favoring a magnetically launched jet, likely launched via the BZ mechanism [D], which extracts the spin energy of the $\mathrm{BH}$ through a magnetic field connecting the $\mathrm{BH}$ event horizon and a remote 
astrophysical load. The bipolar BZ jet power from a BH with mass $M_{\bullet}$ and angular momentum $J$ is [8, Q, [0] $L_{\mathrm{BZ}}=1.7 \times 10^{44} a_{\bullet}^{2} M_{\bullet \bullet}^{2} B_{\bullet}^{2} F\left(a_{\bullet}\right) \mathrm{erg} \mathrm{s}^{-1}$, where $a_{\bullet}=J c /\left(G M_{\bullet}^{2}\right)$ is the BH spin parameter, $M_{\bullet, 6}=M_{\bullet} / 10^{6} M_{\odot}, B_{\bullet}, 6=B_{\bullet} / 10^{6} \mathrm{G}$ and $F\left(a_{\bullet}\right)=\left[\left(1+q^{2}\right) / q^{2}\right][(q+1 / q) \arctan q-1]$, here $q=a_{\bullet} /\left(1+\sqrt{1-a_{\bullet}^{2}}\right)$. It apparently depends on $M_{\bullet}, B_{\bullet}$, and $a_{\bullet}$. However, since an isolated $\mathrm{BH}$ does not carry a magnetic field, $B_{\bullet}$ is closely related to the accretion rate $\dot{M}$ and the radius of the $\mathrm{BH}$, which depends on $M_{\text {. }}$. Combining these dependences, one finds that $L_{\mathrm{BZ}}$ is essentially a function of $\dot{M}$ and $a_{\text {. }}$. More precisely, we assume that magnetic fields in the disk are in close equilibrium with the pressure, $\frac{B_{\mathrm{disk}}^{2}}{8 \pi} \sim \frac{1}{3} a T^{4}$, where the temperature of the disk ( $a_{\bullet}$ - and $r$-dependent) can be found in solution by Page \& Thorne (1974)[W]. We assume that the strength of the magnetic field threading the $\mathrm{BH}$ is comparable to the largest field strength in the disk, i.e. $B_{\bullet} \sim B_{\text {disk }}^{\max }$. For given $\dot{M}$ and $a_{\bullet}$, we solve numerically the temperature profile of the thin disk and identify the radius $r_{\text {peak }}$ where $T$ reaches the maximum. We then calculate $B_{\mathrm{disk}}^{\mathrm{max}}$, which is assigned to $B_{\bullet}$. One then obtains $L_{\mathrm{BZ}}$ once $\dot{M}$ and $a_{\bullet}$ are specified. If one identifies the $\mathrm{BZ}$ mechanism as the jet launching mechanism, the $\mathrm{BH}$ spin parameter can be constrained from the data.

The observed X-ray luminosity $L_{\mathrm{X} \text {,iso }}$ can be connected to the BZ power $L_{\mathrm{BZ}}$ through $\eta L_{\mathrm{BZ}}=$ $f_{b} L_{\mathrm{X} \text {,iso }}$, where $\eta$ is the efficiency of converting BZ power to X-ray radiation, and $f_{b}$ is the beaming factor of the jet. We adopt $\eta \sim 0.5$, motivated by a potentially high radiation efficiency of a magnetically-dominated jet. The parameter $f_{b}$ has a large uncertainty. We apply a statistical method to estimate the range of $f_{b}$ within the TDE framework, i.e., the ratio of all-sky detection rate of Sw J1644+57-like events to the total jetted TDE rate. We thus have $f_{b} \sim \frac{R_{\mathrm{obs}}}{10 \% R_{\mathrm{tot}}} \in\left(4.4 \times 10^{-5}, 5.5 \times\right.$ $\left.10^{-3}\right)$. Noticing $L_{\mathrm{BZ}} \propto \dot{M}$, one can normalize the accretion rate using the total accreted mass based on the observed flux $F_{\mathrm{X}}$ and fluence $S_{\mathrm{X}}$ of the event. Taking the peak flux as example, the peak accretion rate can be estimated as $\dot{M}_{\text {peak }}=\frac{F_{\mathrm{X}}^{\text {peak }}(1+z)}{S_{\mathrm{X}}} \Delta M, \Delta M=f_{\mathrm{m}} M_{*}$ is the mass that falls back to pericenter, which is a fraction $f_{\mathrm{m}}$ of the original mass of the disrupted star $M_{*}$. The mass fraction $f_{\mathrm{m}}$ was assumed to be 0.5 in Rees (1988)[2], which means half of the debris is bound. However, recent numerical simulations suggested a smaller fraction $f_{\mathrm{m}} \simeq 0.1$ [12]. In the following calculation we adopt $f_{\mathrm{m}}=0.1$. The range of $M_{*}$ may be between $0.1 M_{\odot}$ and $10 M_{\odot}$. One can then derive a mass-dependent constraint on $a_{\bullet}$.

Figure Wla presents the constraint on $a_{\bullet}$ for Sw J1644+57 as a function of $M_{*}$. The two boundary lines correspond to two ends of the range of beaming factor of the jet $f_{b}$, with the lower and upper boundaries correspond to $f_{b}=4.4 \times 10^{-5}$ and $f_{b}=5.5 \times 10^{-3}$, respectively. The middle dashed line corresponds to the most probable value $f_{b} \sim 10^{-3}$. One can see that the supermassive BH is demanded to have a moderate to high spin rate. Given the standard stellar initial mass function, the number of low-mass stars is much more abundant than the high-mass stars. If one takes $M_{*}=1 M_{\odot}$, the required range of $a_{\bullet}$ is $(0.51,0.98)$, with the most probably value $a_{\bullet}=0.90$.

A second candidate in the same category, Sw J2058+05 was reported by Cenko et al. (2011), who argued that its observational properties are rather similar to those of Sw J1644+57. The constraint on BH spin for Sw $\mathbf{J} 2058+05$ is shown in Figure $\mathrm{bl}$. We find that the demand for BH spin is even more stringent for this source. For $M_{*}=1 M_{\odot}$, the required range of $a_{\bullet}$ is $(0.81,0.998)$, with the most probably value $a_{\bullet}=0.99$.

For such events, since the star initially has no knowledge about the $\mathrm{BH}$ spin orientation before 

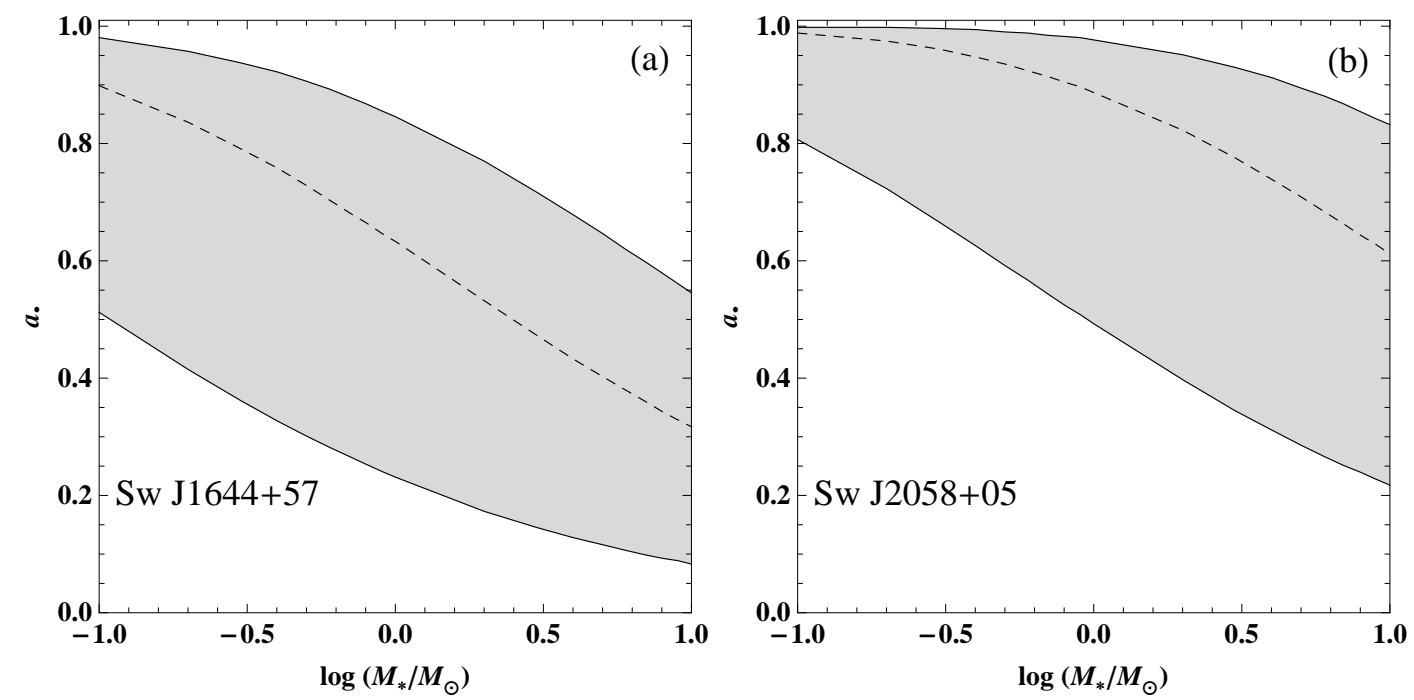

Figure 1: Parameter space of $M_{*}-a_{\bullet}$ for Sw J1644+57 (left panel) and Sw J2058+05 (right panel). The shaded region indicate the range for BH spin bracketed by $f_{b}=4.4 \times 10^{-5}$ (lower) and $f_{b}=5.5 \times 10^{-3}$ (upper). The dashed line corresponds to the most probable value $f_{b}=10^{-3}$.

being disrupted and accreted, it is most likely that the initial stellar orbit is mis-aligned with the equatorial plane of the spinning BH. A natural expectation is that at least the outer part of the accretion disk is also misaligned. The tilted disk surrounding a spinning $\mathrm{BH}$ is subject to the Lense-Thirring (LT hereafter) torque [ㅍ] . The combined action of the LT torque and the internal viscosity of the accretion disk would lead to a twisted and warped disk, with the inner part of the disk bent towards the $\mathrm{BH}$ equatorial plane due to the frame-dragging effect. This is known as the Bardeen-Petterson effect [[2]]. The relativistic jet launched from the spinning SMBH would undergo precession.

Besides this theoretical motivation, some curious observational facts also point toward the possibility of a warped disk. More importantly, a closer inspection suggests that the late X-ray light curve show dips. A natural mechanism to account for these dips would be a precessing jet, possibly caused by a warped disk [ए]3].

We investigate this independently using SFC method [미]. The SFC method is a signal processing algorithm which stepwisely filters signals above a frequency, and looks for a correlation between the light curves of adjacent filtered light curves. We performed an analysis of the entire light curve of Sw J1644+57. Since the early light curve displays many erratic, bright X-ray flares, the signal is dominated by these high-frequency components and the 2.7 day component cannot be identified. If we remove the early data (say, the first two days), the 2.7 day dip clearly shows up (Figure 2). Our analysis suggests that, consistent with the results of Burrows et al. (2011) and Saxton et al. (2012)[[2]], that the 530 day X-ray light curve of Sw J1644+57 has a quasi-periodic signal with a period of roughly 2.7 days.

\section{TDE with an off-beam relativistic jet}

IGR J12580+0134 was discovered by Integral [23] during January 2-11, 2011, with a position 


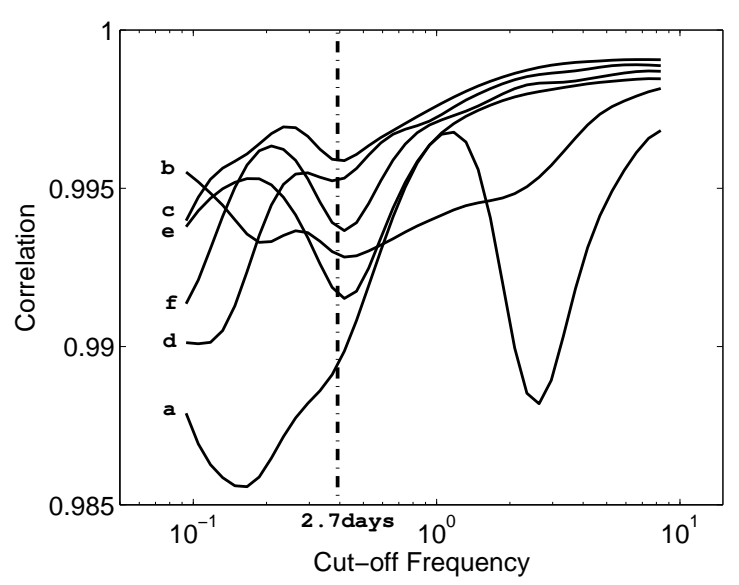

Figure 2: The stepwise filter correlation (SFC) analysis of the X-ray lightucrve of Sw J1644+57. The curve (a) is the result for the entire lightcurve, curve (b), (c), (d), (e) and (f) are the analyses by manually removing the first 2, 5, 10, 15, and 20 days of the data, respectively. A dip corresponding to $\sim 2.7$ days is evident once the first 2-day of data is removed.

consistent with that of a nearby spiral galaxy NGC 4845. Swift/XRT and XMM-Newton observations started a few days later, confirmed the association of the transient with the nucleus of the galaxy.

The data suggested that the source resulted from a TDE of a super-Jupiter by the central SMBH in NGC 4845 [24]. The X-ray lightcurve after the peak is consistent with the $t^{-5 / 3}$ decay law, as expected by the simple TDE picture.

The comparison of the X-ray emissions between Sw J1644 +57 (scaled down by a factor of 1/300; gray points) and IGR J12580+0134 (red, in energy band $17.380 \mathrm{keV}$ ) is shown in Figure 3a. One can see that the observed emissions are substantially lower than what would be expected from an on-beam jet. The peak of the observed $17.3-80 \mathrm{keV}$ luminosity is $\sim 1.5 \times 10^{42} \mathrm{erg} \mathrm{s}^{-1}$ for IGR J12580+0134, and is $\sim 3.25 \times 10^{48} \mathrm{erg} \mathrm{s}^{-1}$ for Sw J1644+57. Therefore, the observed peak luminosity of IGR J12580+0134 is 7200 times fainter than what might be expected for an on-beam jet. The jet associated with this TDE must be off-beam [1]6], as shown in Figure $3 b$ with a cartoon representing the emission structure of the source.

Radio emission from IGR J12580+0134 has been detected by JVLA at 1.57 and $6 \mathrm{GHz}$ about one year after the X-ray peak [25]. The energy spectra, polarization properties, and the time evolution of the radio emission suggest a self-absorbed synchrotron emission origin from an expanding radio lobe powered by the jet associated with the TDE. We studied the jet dynamics in detail with a numerical model of the jet evolution [26] and external shock emission [27]. The model is used to fit the lightcurves and spectra in radio [28]. Figure 4 shows an illustration of the model expectations compared with the data. The radio light curves and multiband spectra can be well explained by the synchrotron emission from the external shock of the jet (Figure 4). The data were not obtained simultaneously for 1.57 and $6.0 \mathrm{GHz}$. The radio spectra were actually obtained by interpolating or extrapolating the data to three epochs [25], i.e., 2011 December 30 (T1), 2012 February 24 (T2), and 2012 July 13 (T3). This may account for the deviation of the $6 \mathrm{GHz}$ flux at T3 from the model prediction. 

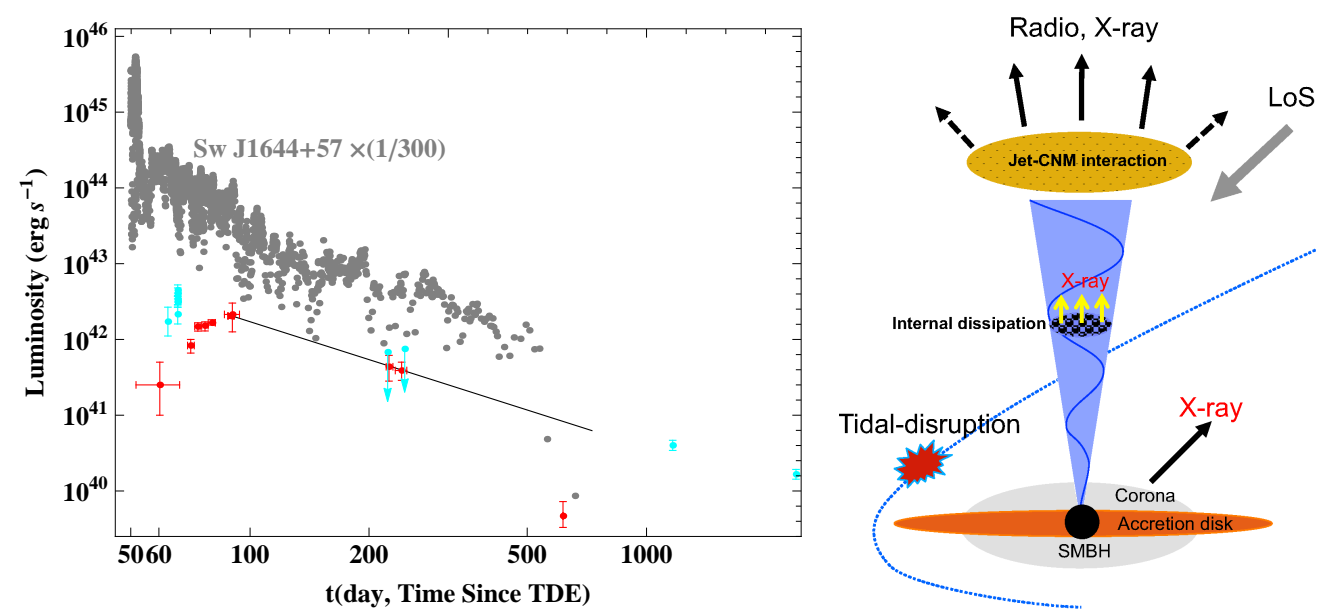

Figure 3: Left panel: The X-ray emission in IGR J12580+0134 (red) compared with the X-ray data of Sw J1644+57 scaled down by a factor of 300 (gray). The observational data of IGR J12580+0134 (17.3-80 keV) are adopted from Nikolajuk \& Walter (2013). The solid line shows the $t^{-5 / 3}$ power law fit. For comparison, we also show a ROSAT candidate in NGC 5905 (in $0.1-2 \mathrm{keV}$ ) with cyan (the data were taken from Li et al. 2002), which is a typical non-jetted TDE [29]. This suggests that the hard X-ray emission may be dominated by that from disk/corona. The contribution from internal dissipation should fall below it due to the strong suppression from an off-beam jet. Right panel: A schematic illustration of the model, consisting of an accretion disk around the SMBH in NGC 4845, and an off-beam relativistic jet. "LoS" denotes the direction of the line-of-sight. X-ray emission can be expected from the disk, corona, and in the jet (through internal dissipation and jet-CNM interaction). The jet components are strongly suppressed at early times due to the large Lorentz factor and view angle. The observed hard X-ray emission comes primarily from an accretion disk and its associated corona near the SMBH. Bright radio emission, originated from the external shock due to interaction between the jet and the CNM, will be observed when the jet enters the Newtonian phase (emission shown with the dashed arrows).

Our modeling, therefore, establishes IGR J12580+0134 as the first TDE with an off-beam relativistic jet.

\section{Conclusions}

Sw J1644+57 and Sw J2058+05 are the first two proto-type objects in the newly identified astrophysical phenomenon, namely, a relativistic jet associated with a TDE from a supermassive BH. A straightforward question is why only some TDEs launch jets. We found that both events need to invoke a $\mathrm{BH}$ with a moderate to rapid spin in order to interpret the observations: the most probably values are $a_{\bullet}(\mathrm{J} 1644)=0.63,0.90$ and $a_{\bullet}(\mathrm{J} 2058)=0.89,0.99$ for $M_{*}=1,0.1 M_{\odot}$, respectively. We therefore suggest that $\mathrm{BH}$ spin is the key factor behind the Sw J1944+57-like events.

We proposed that the Sw J1644+57 jet launched from a SMBH precesses. This comes from two independent arguments. First, a tidally disrupted star should not have knowledge about the spin direction of the $\mathrm{BH}$ before disruption, so that the original stellar orbit very likely has an inclination with respect to the equatorial plane of the $\mathrm{BH}$. A rapidly spinning $\mathrm{BH}[\mathrm{W}]$ tends to distort the accretion flow and warp the accretion disk due to strong LT frame dragging. This 

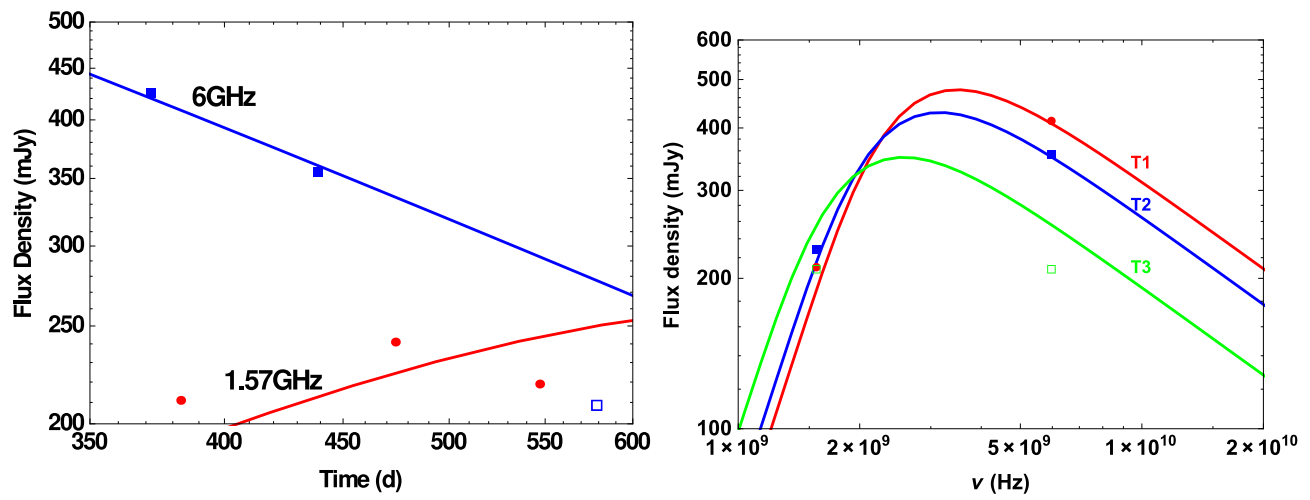

Figure 4: Left panel: Radio lightcurves of IGR J12580+0134 extending to $t \simeq 600$ days at $1.57 \mathrm{GHz}$ (red) and $6 \mathrm{GHz}$ (blue), respectively. The observational data (filled symbol) are adopted from Irwin et al. (2015). The $6 \mathrm{GHz}$ flux at $\mathbf{5 7 9}$ days (blue open square) is an extrapolation to the observed data. Right panel: Multi-frequency radio spectral distributions of IGR J12580+0134 at $t \simeq 383$ (T1, red), 439 (T2, blue), and 579 (T3, green) days. Note that the data (squares) were not obtained simultaneously for different bands. They were interpolated or extrapolated to these three epochs (Irwin et al. 2015). This may explain the deviation of the $6 \mathrm{GHz}$ flux at $\mathrm{T} 3$ from the model prediction.

would naturally result in a precessing jet if the accretion rate is low enough. Second, observational evidence hint towards a precessing jet: the rough 2.7-day periodicity and the noticeable lightcurve dips. We performed a SFC analysis to the observed X-ray lightcurve, and confirmed the 2.7-day rough period.

The nearby TDE IGR J12580+0134, discovered in X-rays by Integral and then detected in radio by JVLA, likely launched a relativistic jet. Taking Sw J1666+57 as a template, we find that the expected internal jet emission would outshine the observed flux greatly. This suggests that the jet is off-beam, suggesting IGR J12580+0134 as the first TDE with an off-beam relativistic jet.

\section{References}

[1] Magorrian, J. \& Tremaine, S. 1999, MNRAS, 309, 447

[2] Rees, M. J. 1988, Nature, 333, 523

[3] Kobayashi, S., Laguna, P., Phinney, E. S. \& Mészáros, P. 2004, ApJ, 615, 855

[4] Burrows, D. N., Kennea, J. A., Ghisellini, G., et al. 2011, Nature, 476, 421

[5] Zauderer, B. A., Berger, E., Soderberg, A. M., et al. 2011, Nature, 476, 425

[6] Blandford, R. D., \& Znajek, R. L. 1977, MNRAS, 179, 433

[7] Lei, W. H., \& Zhang, B. 2011, ApJ, 740, L27

[8] Wang, D. X, Xiao, K., \& Lei, W. H., 2002, MNRAS, 335, 655

[9] McKinney, J. C. 2005, ApJ, 630, L5

[10] Komissarov, S. S., \& Barkov, M. V. 2010, MNRAS, 402, L25

[11] Page, D. N., \& Thorne, K. S. 1974, ApJ, 191, 499

[12] Ayal, S., Livio, M., \& Piran, T. 2000, ApJ, 545, L143 
[13] Lei, W. H., Zhang, B., \& Gao, H. 2013, ApJ, 762, 98

[14] Cenko, S. B., Krimm, H. A., Horesh, A., et al. 2012, ApJ, 753, 77

[15] Brown, G. C., Levan, A. J., Stanway, E. R., et al. 2015, arXiv:1507.03582

[16] Lei, W. H., Yuan, Q., Zhang, B., \& Wang, D. 2016,ApJ, 816, 20

[17] Gehrels, N. et al. 2004, ApJ, 611, 1005

[18] Levan, A. J., Tanvir, N. R., Cenko, S. B., et al. 2011, Science, 333, 199

[19] Lense, J., \& Thirring, H. 1918, Phys. Z., 19, 156

[20] Bardeen J. M., Press W. H., \& Teukolsky S. A. 1972, ApJ, 178, 347

[21] Gao, H., Zhang, B. B., \& Zhang, B., ApJ, 748, 134 (2012)

[22] Saxton, C. J., Soria, R., Wu, K., \& Kuin, N. P. M., MNRAS, 422, 1625 (2012)

[23] Walter, R. et al. 2011, ATel, 3108

[24] Nikolajuk, M., \& Walter, R. 2013, A\&A, 552, A75

[25] Irwin, J. A., Henriksen, R. N., Krause, M., Wang, Q. D., Wiegert, T., Murphy, E. J., Heald, G., \& Perlman, E. 2015, ApJ, 809, 172

[26] Huang, Y. F., Gou, L. J., Dai, Z. G., \& Lu, T. 2000, ApJ, 543, 90

[27] Gao, H., Lei, W. H., Zou, Y. C., Wu, X. F., \& Zhang, B. 2013, New Astron. Rev. , 57, 141

[28] Wang, J. Z., Lei, W. H., Wang, D. X., Zou, Y. C., Zhang, B., Gao, H., \& Huang, C. Y. 2014, ApJ, 788, 32

[29] Li, L. X., Narayan, R., \& Menou, K. 2002, ApJ, 576, 753 